\title{
Value of Longitudinal Strain to Identify Wild-Type Transthyretin Amyloidosis in Patients With Aortic Stenosis
}

\author{
Guillaume Robin, MD; Thomas Cognet, MD; Frédéric Bouisset, MD; Eve Cariou, MD; \\ Simon Méjean, MD; Sarah Pradel, MD; Magali Colombat, MD, PhD; \\ Michel Galinier, MD, PhD; Didier Carrié, MD, PhD; Olivier Lairez, MD, PhD \\ on behalf of the Toulouse Amyloidosis Research Network Collaborators
}

\begin{abstract}
Background: Wild-type transthyretin-related amyloidosis (ATTRwt) and degenerative aortic stenosis (AS) are both age-related. Diagnosis of cardiac amyloidosis (CA) among patients with AS may be difficult due to overlapping morphological and functional criteria. The aim of this study was to describe an echocardiographic longitudinal strain (LS) pattern among patients with AS with and without ATTRwt.

Methods and Results: Patients who have AS with ATTRwt $(n=30)$, AS without ATTRwt $(n=50)$ and ATTRwt without AS $(n=31)$ underwent two-dimensional speckle-tracking echocardiography. Transthyretin CA was based on positive bone scintigraphy without monoclonal gammopathy. All patients showed a gradual decrease in LS from the base to the apex resulting in a decrease of the global LS. A cut-off value of 1.0 for relative apical LS (average apical LS/[average basal LS+mid-LS]) was sensitive (88\%) but less specific (68\%) in differentiating ATTRwt among patients with severe AS. The best cut-off value for relative apical LS for identifying patients with ATTRwt among the whole population was 0.9 (sensitivity $74 \%$, specificity $66 \%$ ); however, $35 \%, 25 \%$ and $11 \%$ of patients who have ATTRwt without AS, with moderate AS and with severe AS, respectively, did not reach this threshold.
\end{abstract}

Conclusions: A decrease of global and relative apical LS is common in patients with AS, even in the absence of ATTRwt. ATTRwt CA can be present even in the absence of relative apical sparing of LS.

Key Words: Aortic stenosis; Cardiac amyloidosis; Echocardiography; Longitudinal strain

$\mathbf{S}$ evere degenerative aortic stenosis (AS) is common in the elderly, affecting $2.9 \%$ of individuals aged $>75$ years. ${ }^{1}$ Early valve replacement is strongly recommended in all symptomatic patients with severe AS because of their dismal prognosis if treated medically. Exceptions are patients in whom severe comorbidities make it unlikely that the intervention will improve their quality of life or survival. ${ }^{2}$ Consequently, the selection of patients who will benefit from invasive treatment of severe AS requires the screening for comorbidities, especially in patients with low flow, low gradient AS and preserved ejection fraction who are likely to be referred for aortic valve replacement despite a higher risk of mortality. ${ }^{3}$ Wild-type transthyretin related amyloidosis (ATTRwt) is also a disorder of aging individuals and has been reported in $6 \%$ among patients with AS aged $>65$ years undergoing surgical aortic valve replacement ${ }^{4}$ and in $16 \%$ of patients with severe calcific AS undergoing transcatheter aortic valve replacement. ${ }^{5}$ Mildto-severe myocardial amyloidosis has been found upon pathology of autopsy cases in $29 \%$ after transcatheter aortic valve implantation. ${ }^{6}$ Occult ATTRwt cardiac amyloidosis in patients with severe AS has been associated with a severe phenotype of low flow, low gradient and a poor outcome. ${ }^{4,5}$ The poorer prognosis of patients with both AS and ATTRwt amyloidosis, along with contemporary therapeutic options for ATTR amyloidosis, ${ }^{7-9}$ make the detection of cardiac amyloidosis extremely important in patients with symptomatic AS.

The heart failure profile of patients suffering from AS and cardiac amyloidosis may be similar. Adaptive left ventricular hypertrophy caused by chronic pressure overload may be similar to a classic description of cardiac amyloidosis regarding left ventricular hypertrophy or diastolic dysfunction. Non-invasive diagnosis of cardiac amyloidosis

Received October 13, 2020; revised manuscript received March 17, 2021; accepted March 19, 2021; J-STAGE Advance Publication released online May 11, 2021 Time for primary review: 19 days

Department of Cardiology, Rangueil University Hospital, Toulouse (G.R., T.C., F.B., E.C., S.M., S.P., M.G., D.C., O.L.); Cardiac Imaging Center (G.R., T.C., E.C., S.M., S.P., M.G., D.C., O.L.), Department of Nuclear Medicine (T.C., S.M., O.L.), Toulouse University Hospital, Toulouse; Department of Pathology, IUCT Oncopôle, Toulouse (M.C.); and Medical School, Toulouse III Paul Sabatier University, Toulouse (M.G., D.C., O.L.), France

Mailing address: Olivier Lairez, MD, PhD, Department of Cardiology, Rangueil University Hospital, 1, avenue Jean Poulhès, TSA 50032, 31059 Toulouse Cedex 9, France. E-mail: lairez@gmail.com

All rights are reserved to the Japanese Circulation Society. For permissions, please e-mail: cj@j-circ.or.jp

ISSN-1346-9843 
in patients referred for AS treatment may be challenging. Global longitudinal strain (LS) may be helpful to suspect amyloidosis in the presence of left ventricular hypertrophy. ${ }^{10}$ The relative 'apical sparing' pattern of LS appears to be an accurate and reproducible method to differentiate cardiac amyloidosis from other causes of left ventricular hypertrophy. ${ }^{11}$ It has been demonstrated that visual assessment of relative apical sparing pattern is a useful and powerful tool for diagnosing cardiac amyloidosis in borderline or mildly increased left ventricular wall thickness. ${ }^{12}$ It remains unclear, however, whether the relative 'apical sparing' pattern of LS is still accurate and should be used in patients with AS.

Therefore, the aim of our study was to explore the pattern of LS among patients with AS with and without ATTRwt amyloidosis.

\section{Methods}

\section{Study Population}

Patients referred to the Rangueil University Hospital echocardiography laboratory for severe AS (i.e., aortic valve area $\leq 1.0 \mathrm{~cm}^{2}$ ) from November 2015 and November 2017 were included in this case-control study. All patients underwent $99 \mathrm{mTc}$-hydroxymethylene-diphosphonate

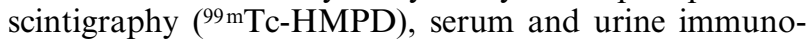
fixation and serum-free light chain assay. Patients with grades 2 or $3{ }^{99} \mathrm{~m}$ Tc-HMPD scintigraphy without monoclonal gammopathy were diagnosed for ATTRwt cardiac amyloidosis. ${ }^{13}$ Patients referred to the Rangueil University Hospital Department of Nuclear Medicine for the presumptive diagnosis of ATTRwt cardiac amyloidosis with grades 2 or $3^{99 \mathrm{~m}} \mathrm{Tc}-\mathrm{HMPD}$ scintigraphy without monoclonal gammopathy during the same period were included in the study.

Patients were divided into 4 groups according to the presence of moderate or severe AS and cardiac amyloidosis as follow: 31 patients who had ATTRwt without AS (group 1), 12 patients with moderate AS and concomitant ATTRwt (group 2), 18 patients with severe AS and concomitant ATTRwt (group 3), and 50 patients with severe AS without ATTRwt (group 4).

All patients underwent rest echocardiography. Three patients referred for severe AS were excluded because of a poor acoustic window. DNA analysis with transthyretin gene sequencing was performed for patients with ATTR amyloidosis to distinguish hereditary from ATTRwt.

The investigation conforms with the principles outlined in the Declaration of Helsinki. All patients were informed of the design of the study and gave their consent. The study was approved by our institutional review board (ethics committee for research of the University Hospital of Toulouse - reference number: 02-815).

\section{Echocardiography}

All patients underwent a transthoracic echocardiography at the inclusion with a commercially available ultrasound Vivid E9 system (GE Vingmed Ultrasound AS, Horten, Norway) using a 2,5 MHz transducer. A complete M-mode and two-dimensional gray scale echocardiography, including the 3 standard apical views (4, 3 and 2 chamber) using high frame rates $(>60$ frames/s), were performed for each patient. All echocardiographic data were synchronized to the electrocardiogram, and acquisition was performed during breath hold.
Image analysis was independently performed by a single blinded observer unaware of clinical and scintigraphic data (G.R.). For offline two-dimensional strain imaging, the offline EchoPAC software version 201 (GE Vingmed Ultrasound AS, Horten, Norway) was used. The tracking quality was considered as correct for all patients. The following measurements were prospectively recorded according to the American Society of Echocardiography (ASE) and the European Association of Cardiovascular Imaging (EACVI) guidelines:14 LV end-diastolic and -systolic volumes and ejection fraction using Simpson's biplane method from apical 2- and 4-chamber views, left atrial volume index using the biplane area length formula, end-diastolic interventricular septal and posterior wall thickness, and end-diastolic left ventricular diameter. Wall motion score index was calculated according to the ASE and EACVI guidelines using a 17-segment model and the scoring system: (1) normal; (2) hypokinetic; (3) akinetic; and (4) dyskinetic. ${ }^{14}$ Diastolic parameters, including diastolic mitral inflow velocity pattern, medial and lateral mitral annular diastolic velocities (Ea) were also recorded according to ASE/EACVI guidelines. ${ }^{15}$ All Doppler measurements were made over 3 cardiac cycles and averaged. In patients with atrial fibrillation, the data were averaged over 5 cardiac cycles. Diagnostic of moderate (i.e., aortic valve area between 1.0 and $1.5 \mathrm{~cm}^{2}$ ) and severe (i.e., aortic valve area $<1.0 \mathrm{~cm}^{2}$ ) AS was established from the ASE/EACVI guidelines. ${ }^{16}$ Longitudinal myocardial strain was calculated using speckle tracking from 2D gray-scale images and the automated function imaging technique. ${ }^{17}$ The LV endocardium was manually identified, and tissue speckles were automatically tracked frame by frame throughout the cardiac cycle. Strain values from all segments were averaged to obtain a global LS value. The strain values for the 6 basal, 6 mid and 4 apical segments of the LV were averaged to obtain 3 'regional' LS values. The apex-to-base gradient in regional LS was examined using a relative apical LS calculated as: relative apical LS = average apical LS / (average basal LS + average mid LS). ${ }^{11}$

\section{$99 \mathrm{mTC}$-Hydroxymethylene-Diphosphonate Scintigraphy}

All images were acquired on a hybrid dual-head SPECT/CT system (Symbia T6; Siemens Healthcare, Erlangen, Germany) equipped with low-energy, high-resolution collimators $3 \mathrm{~h}$ after injection of $740 \mathrm{MBq}$ of ${ }^{99 \mathrm{~m} T \mathrm{c}-\mathrm{HMDP}}$ intravenously. Acquisitions consisted of a whole-body scan $(20 \mathrm{~cm} / \mathrm{min})$ in the anterior and posterior views, followed by a tomographic acquisition, consisting of a standard helical CT $(120 \mathrm{kV}, 100 \mathrm{mAs}$, slice thickness $2.0 \mathrm{~mm}, 512 \times 512$ matrix) for attenuation correction, followed by a SPECT covering the thorax (120 projections, 20 s/projection, $128 \times 128$ matrix).

Image analysis was independently performed by a single blinded observer unaware of clinical and echocardiographic data (T.C.). Planar images were image analyzed using on the GE Xeleris workstation, and visual scoring of cardiac retention was defined as described previously:18 score 0 , absent cardiac uptake and normal bone uptake; score 1, mild cardiac uptake, inferior to bone uptake; score 2 , moderate cardiac uptake associated with attenuated bone uptake; or score 3, strong cardiac uptake with mild/absent bone uptake. ${ }^{99} \mathrm{~m} \mathrm{Tc}-\mathrm{HMDP}$ was considered as suggestive of transthyretin cardiac amyloidosis when the visual score was $\geq 2$. 
Table 1. Baseline Characteristics According to the Presence of Aortic Stenosis and/or Wild-Type Transthyretin Amyloidosis

\begin{tabular}{|c|c|c|c|c|c|}
\hline & $\underset{(n=111)}{\text { All }}$ & $\begin{array}{c}\text { Group 1 } \\
\text { AS-/ATTR+ } \\
(n=31)\end{array}$ & $\begin{array}{c}\text { Group 2 } \\
\text { mAS+/ATTR+ } \\
(n=12)\end{array}$ & $\begin{array}{c}\text { Group } 3 \\
\text { sAS+/ATTR+ } \\
(n=18)\end{array}$ & $\begin{array}{c}\text { Group } 4 \\
\text { sAS+/ATTR- } \\
(n=50)\end{array}$ \\
\hline \multicolumn{6}{|l|}{ Clinical data } \\
\hline Age, years & $79 \pm 12$ & $79 \pm 11$ & $82 \pm 6$ & $86 \pm 4$ & $79 \pm 15$ \\
\hline Gender, male, n (\%) & $82(74)$ & $27(87)$ & $12(100)$ & $16(89)$ & $27(54)$ \\
\hline $\mathrm{BMI}, \mathrm{kg} / \mathrm{m}^{2}$ & $26 \pm 4$ & $25 \pm 3$ & $26 \pm 3$ & $25 \pm 3$ & $27 \pm 5$ \\
\hline Systolic arterial blood pressure, $\mathrm{mmHg}$ & $131 \pm 19$ & $123 \pm 17$ & $126 \pm 21$ & $129 \pm 13$ & $137 \pm 21$ \\
\hline Diastolic arterial blood pressure, $\mathrm{mmHg}$ & $75 \pm 12$ & $74 \pm 13$ & $74 \pm 16$ & $76 \pm 11$ & $74 \pm 11$ \\
\hline \multicolumn{6}{|l|}{ Cardiovascular risk factors, n (\%) } \\
\hline Diabetes mellitus & $22(20)$ & $3(10)$ & $4(33)$ & $4(22)$ & $11(22)$ \\
\hline Arterial hypertension & $53(48)$ & $11(35)$ & $12(100)$ & $14(78)$ & $25(50)$ \\
\hline Dyslipidemia & $43(39)$ & $10(32)$ & $5(42)$ & $8(44)$ & $20(40)$ \\
\hline Current smoker & $23(21)$ & $7(23)$ & $2(17)$ & $5(28)$ & $9(18)$ \\
\hline \multicolumn{6}{|l|}{ Personal history of CAD, $\mathrm{n}(\%)$} \\
\hline Myocardial infarction scar & $8(7)$ & $1(3)$ & $1(8)$ & $1(6)$ & $5(10)$ \\
\hline Coronary artery disease & $46(41)$ & $7(23)$ & $5(42)$ & $7(39)$ & $27(54)$ \\
\hline \multicolumn{6}{|l|}{ NYHA Functional Class, $n$ (\%) } \\
\hline I & $10(9)$ & $4(13)$ & $2(17)$ & $0(0)$ & $4(8)$ \\
\hline II & $65(59)$ & $20(65)$ & $5(42)$ & $8(44)$ & $32(64)$ \\
\hline III & $23(21)$ & $6(19)$ & $2(17)$ & $7(39)$ & $8(16)$ \\
\hline IV & $11(10)$ & $1(3)$ & $2(17)$ & $3(17)$ & $5(10)$ \\
\hline Mean & $2.3 \pm 0.8$ & $2.1 \pm 0.7$ & $2.2 \pm 1.2$ & $2.7 \pm 0.8$ & $2.3 \pm 0.8$ \\
\hline \multicolumn{6}{|l|}{ Symptoms at admission, $\mathbf{n}(\%)$} \\
\hline Pulmonary oedema & $9(8)$ & $1(3)$ & $1(8)$ & $1(6)$ & $6(12)$ \\
\hline Congestive heart failure & $34(31)$ & $18(58)$ & $4(33)$ & $6(33)$ & $6(12)$ \\
\hline Chest pain & $8(7)$ & $1(3)$ & $0(0)$ & $1(6)$ & $6(12)$ \\
\hline Syncope & $9(8)$ & $4(13)$ & $1(8)$ & $1(6)$ & $3(6)$ \\
\hline \multicolumn{6}{|l|}{ ECG, $n(\%)$} \\
\hline Atrial fibrillation & $42(38)$ & $15(48)$ & $9(75)$ & 7 (39) & $11(22)$ \\
\hline Wide QRS complex & $31(28)$ & $5(16)$ & $6(50)$ & $8(44)$ & $12(24)$ \\
\hline 1 st degree AV block & $14(13)$ & $0(0)$ & $2(17)$ & $5(28)$ & $7(14)$ \\
\hline Low voltage & $5(5)$ & $4(13)$ & $0(0)$ & $1(6)$ & $0(0)$ \\
\hline \multicolumn{6}{|l|}{ Biology } \\
\hline NT pro BNP, pg/mL & $\begin{array}{c}2,331 \\
{[930-5,275]}\end{array}$ & $\begin{array}{c}1,103 \\
{[2,057-5,200]}\end{array}$ & $\begin{array}{c}2,000 \\
{[4,500-12,300]}\end{array}$ & $\begin{array}{c}2,125 \\
{[3,100-4,600]}\end{array}$ & $\begin{array}{c}790 \\
{[1,500-4,925]}\end{array}$ \\
\hline Troponin $\mathrm{T}, \mathrm{ng} / \mathrm{mL}$ & $\begin{array}{c}48 \\
{[19-94]}\end{array}$ & $\begin{array}{c}40 \\
{[7-108]}\end{array}$ & $\begin{array}{c}69 \\
{[52-114]}\end{array}$ & $\begin{array}{c}58 \\
{[22-80]}\end{array}$ & $\begin{array}{c}15 \\
{[39-120]}\end{array}$ \\
\hline GFR, mL/min/kg & $51 \pm 21$ & $50 \pm 20$ & $41 \pm 23$ & $49 \pm 18$ & $54 \pm 21$ \\
\hline \multicolumn{6}{|l|}{ Medications } \\
\hline Furosemide, $\mathrm{n}(\%)$ & $65(59)$ & $18(58)$ & $7(58)$ & $3(17)$ & $27(54)$ \\
\hline Mineralocorticoid receptor antagonists & $18(16)$ & $9(29)$ & $1(8)$ & $2(11)$ & $6(12)$ \\
\hline ACEI/ARB, $n(\%)$ & $40(36)$ & $8(26)$ & $4(33)$ & 7 (39) & $21(42)$ \\
\hline Amiodarone & $26(23)$ & $12(39)$ & $4(33)$ & $3(17)$ & $7(14)$ \\
\hline Vitamin K-antagonists & $47(42)$ & $16(52)$ & $6(50)$ & 7 (39) & $18(36)$ \\
\hline Direct oral anticoagulants & $20(18)$ & $9(29)$ & $3(25)$ & $5(28)$ & $3(6)$ \\
\hline Aspirin & $51(46)$ & $8(26)$ & $5(42)$ & $9(50)$ & $29(58)$ \\
\hline$\beta$-blockers & $44(40)$ & $6(19)$ & $2(17)$ & $10(55)$ & $26(52)$ \\
\hline ND calcium channel blockers & $11(10)$ & $0(0)$ & $0(0)$ & $4(22)$ & $7(14)$ \\
\hline
\end{tabular}

Data are presented as mean \pm SD or $n(\%)$. sAS/ATTRwt-, severe aortic stenosis without wild-type transthyretin amyloidosis; No AS/ATTRwt+, wild-type transthyretin amyloidosis without aortic stenosis; mAS/ATTRwt+, wild-type transthyretin amyloidosis with moderate aortic stenosis; sAS/ATTRwt+, wild-type transthyretin amyloidosis with severe aortic stenosis; ACEl, angiotensin-converting enzyme inhibitors; ARB, angiotensin II receptor blockers; BMI, body mass index; CAD, coronary artery disease; ECG, electrocardiogram; GFR, glomerular filtration rate; ND, nondihydropyridine; NYHA, New York Heart Association. 


\begin{tabular}{|c|c|c|c|c|c|c|c|}
\hline & \multirow{2}{*}{$P$ value } & \multicolumn{6}{|c|}{ Post-hoc analysis } \\
\hline & & 1 vs. 2 & 2 vs. 3 & 3 vs. 4 & 1 vs. 3 & 1 vs. 4 & 2 vs. 4 \\
\hline \multicolumn{8}{|l|}{ Clinical data } \\
\hline Age, years & 0.001 & 0.101 & 0.056 & 0.004 & $<0.001$ & 0.103 & 0.504 \\
\hline Gender, male, n (\%) & $<0.001$ & 0.255 & 0.352 & 0.007 & 0.616 & 0.002 & 0.002 \\
\hline $\mathrm{BMI}, \mathrm{kg} / \mathrm{m}^{2}$ & 0.698 & & & & & & \\
\hline Systolic arterial blood pressure, $\mathrm{mmHg}$ & 0.024 & 0.727 & 0.674 & 0.097 & 0.318 & 0.005 & 0.114 \\
\hline Diastolic arterial blood pressure, $\mathrm{mmHg}$ & 0.980 & & & & & & \\
\hline \multicolumn{8}{|l|}{ Cardiovascular risk factors, $\mathrm{n}(\%)$} \\
\hline Diabetes mellitus & 0.303 & & & & & & \\
\hline Arterial hypertension & 0.006 & 0.154 & 0.001 & 0.043 & 0.009 & 0.199 & 0.031 \\
\hline Dyslipidemia & 0.821 & & & & & & \\
\hline Current smoker & 0.828 & & & & & & \\
\hline \multicolumn{8}{|l|}{ Personal history of CAD, $n(\%)$} \\
\hline Myocardial infarction scar & 0.688 & & & & & & \\
\hline Coronary artery disease & 0.040 & 0.190 & 0.588 & 0.184 & 0.186 & 0.004 & 0.304 \\
\hline \multicolumn{8}{|l|}{ NYHA Functional Class, $\mathbf{n}(\%)$} \\
\hline 1 & 0.351 & & & & & & \\
\hline II & 0.267 & & & & & & \\
\hline III & 0.217 & & & & & & \\
\hline IV & 0.379 & & & & & & \\
\hline Mean & 0.064 & & & & & & \\
\hline \multicolumn{8}{|l|}{ Symptoms at admission, $\mathrm{n}(\%)$} \\
\hline Pulmonary oedema & 0.539 & & & & & & \\
\hline Congestive heart failure & $<0.001$ & 0.132 & 0.656 & 0.051 & 0.084 & $<0.001$ & 0.091 \\
\hline Chest pain & 0.326 & & & & & & \\
\hline Syncope & 0.702 & & & & & & \\
\hline \multicolumn{8}{|l|}{ ECG, $n(\%)$} \\
\hline Atrial fibrillation & 0.003 & 0.108 & 0.057 & 0.140 & 0.366 & 0.013 & 0.001 \\
\hline Wide QRS complex & 0.052 & & & & & & \\
\hline 1st degree $\mathrm{AV}$ block & 0.037 & 0.073 & 0.403 & 0.169 & 0.004 & 0.029 & 0.559 \\
\hline Low voltage & 0.045 & 0.255 & 0.600 & 0.014 & 0.386 & 0.019 & - \\
\hline \multicolumn{8}{|l|}{ Biology } \\
\hline NT pro BNP, pg/mL & 0.081 & & & & & & \\
\hline Troponin $\mathrm{T}, \mathrm{ng} / \mathrm{mL}$ & 0.683 & & & & & & \\
\hline GFR, $\mathrm{mL} / \mathrm{min} / \mathrm{kg}$ & 0.227 & & & & & & \\
\hline \multicolumn{8}{|l|}{ Medications } \\
\hline Furosemide, n (\%) & 0.094 & & & & & & \\
\hline Mineralocorticoid receptor antagonists & 0.151 & & & & & & \\
\hline ACEI/ARB, n (\%) & 0.516 & & & & & & \\
\hline Amiodarone & 0.054 & & & & & & \\
\hline Vitamin K-antagonists & 0.514 & & & & & & \\
\hline Direct oral anticoagulants & 0.029 & 0.555 & 0.604 & 0.008 & 0.597 & 0.006 & 0.081 \\
\hline Aspirin & 0.042 & 0.256 & 0.471 & 0.005 & 0.081 & 0.004 & 0.242 \\
\hline$\beta$-blockers & 0.004 & 0.607 & 0.038 & 0.001 & 0.011 & 0.003 & 0.027 \\
\hline ND calcium channel blockers & 0.033 & - & 0.112 & 0.020 & 0.014 & 0.029 & 0.203 \\
\hline
\end{tabular}

\section{Statistical Analysis}

Continuous variables were tested for normal distribution using the Kolmogorov-Smirnov test and expressed as mean \pm standard deviation. Nominal values were expressed as numbers and percentages. Biomarkers were not normally distributed, and results were, therefore, presented as medians with interquartile ranges. The study population was categorized into 4 groups, and group comparisons were made using non-parametric Kruskal-Wallis tests for continuous variables and the Pearson chi-squared test for categorical variables, using Bonferroni corrections for multiple comparisons. The accuracy of echocardiographic parameters for predicting ATTRwt was assessed by computing the areas under the receiver operator characteristic (ROC) curves and the best cut-off value was defined as the point with the highest sum of sensitivity and specificity. 


\begin{tabular}{|c|c|c|c|c|c|}
\hline & $\underset{(n=111)}{\text { All }}$ & $\begin{array}{c}\text { Group } 1 \\
\text { AS-/ATTR+ } \\
(n=31)\end{array}$ & $\begin{array}{c}\text { Group } 2 \\
\text { mAS+/ATTR+ } \\
(n=12)\end{array}$ & $\begin{array}{c}\text { Group 3 } \\
\text { sAS+/ATTR+ } \\
(n=18)\end{array}$ & $\begin{array}{c}\text { Group } 4 \\
\text { sAS+/ATTR- } \\
(n=50)\end{array}$ \\
\hline \multicolumn{6}{|l|}{ General parameters } \\
\hline LVEF, \% & $52 \pm 12$ & $50 \pm 10$ & $49 \pm 9$ & $53 \pm 12$ & $54 \pm 12$ \\
\hline LVEF $<50 \%, n(\%)$ & $37(33)$ & $13(41)$ & $6(50)$ & $5(28)$ & $13(26)$ \\
\hline LV end diastolic volume, $\mathrm{mL} / \mathrm{m}^{2}$ & $51 \pm 18$ & $50 \pm 15$ & $42 \pm 16$ & $46 \pm 19$ & $56 \pm 19$ \\
\hline Stroke volume index, $\mathrm{mL} / \mathrm{m}^{2}$ & $27 \pm 10$ & $30 \pm 12$ & $25 \pm 6$ & $26 \pm 14$ & $27 \pm 8$ \\
\hline LV septal wall thickness, $\mathrm{mm}$ & $16 \pm 4$ & $18 \pm 4$ & $18 \pm 4$ & $17 \pm 3$ & $14 \pm 3$ \\
\hline LV posterior wall thickness, $\mathrm{mm}$ & $18 \pm 10$ & $17 \pm 7$ & $18 \pm 8$ & $24 \pm 16$ & $15 \pm 10$ \\
\hline LV mass index, $\mathrm{g} / \mathrm{m}^{2}$ & $117 \pm 50$ & $118 \pm 36$ & $130 \pm 36$ & $176 \pm 71$ & $96 \pm 37$ \\
\hline Wall motion score index & $21 \pm 7$ & $23 \pm 8$ & $23 \pm 8$ & $21 \pm 8$ & $20 \pm 7$ \\
\hline \multicolumn{6}{|l|}{ Transmitral flow (\%) } \\
\hline Type 1 & $40(36)$ & $8(26)$ & $2(17)$ & $4(22)$ & $26(52)$ \\
\hline Type 2 & $22(20)$ & $8(26)$ & $2(17)$ & $4(22)$ & $8(16)$ \\
\hline Type 3/Exclusive E wave & $49(44)$ & $15(48)$ & $8(66)$ & $10(56)$ & $16(32)$ \\
\hline Lateral e' wave, $\mathrm{cm} / \mathrm{s}$ & $6 \pm 2$ & $6 \pm 2$ & $5 \pm 2$ & $5 \pm 2$ & $6 \pm 3$ \\
\hline Left atrial volume, $\mathrm{mL} / \mathrm{m}^{2}$ & $46 \pm 17$ & $45 \pm 15$ & $52 \pm 14$ & $46 \pm 16$ & $45 \pm 20$ \\
\hline \multicolumn{6}{|l|}{ Aortic valvular parameters } \\
\hline Mean transvalvular gradient, $\mathrm{mmHg}$ & $33 \pm 21$ & $4 \pm 2$ & $20 \pm 6$ & $38 \pm 16$ & $47 \pm 15$ \\
\hline Mean transvalvular gradient $<40 \mathrm{mmHg}, \mathrm{n}(\%)$ & $65(59)$ & $31(100)$ & $12(100)$ & $10(55)$ & $12(24)$ \\
\hline AVA, $\mathrm{cm}^{2}$ & $1.2 \pm 0.9$ & $2.7 \pm 0.8$ & $1.3 \pm 0.1$ & $0.8 \pm 0.2$ & $0.8 \pm 0.2$ \\
\hline iAVA, $\mathrm{cm}^{2} / \mathrm{m}^{2}$ & $0.7 \pm 0.5$ & $1.5 \pm 0.5$ & $0.7 \pm 0.1$ & $0.4 \pm 0.1$ & $0.4 \pm 0.1$ \\
\hline Transvalvular peak velocity, $\mathrm{m} / \mathrm{s}$ & $3.3 \pm 1.3$ & $1.3 \pm 0.3$ & $2.8 \pm 0.5$ & $3.9 \pm 0.8$ & $4.2 \pm 0.6$ \\
\hline Aortic valve velocity ratio & $0.33 \pm 0.23$ & $0.73 \pm 0.18$ & $0.31 \pm 0.07$ & $0.20 \pm 0.05$ & $0.21 \pm 0.06$ \\
\hline \multicolumn{6}{|l|}{ Longitudinal strain parameters } \\
\hline Global longitudinal strain, \% & $-12 \pm 4$ & $-11 \pm 3$ & $-10 \pm 2$ & $-10 \pm 4$ & $-14 \pm 4$ \\
\hline Mean basal longitudinal strain, $\%$ & $-7 \pm 5$ & $-5 \pm 4$ & $-4 \pm 3$ & $-4 \pm 4$ & $-9 \pm 6$ \\
\hline Mean mid longitudinal strain, \% & $-11 \pm 5$ & $-10 \pm 3$ & $-9 \pm 3$ & $-9 \pm 4$ & $-13 \pm 5$ \\
\hline Mean apical longitudinal strain, \% & $-18 \pm 6$ & $-16 \pm 4$ & $-16 \pm 3$ & $-18 \pm 5$ & $-19 \pm 6$ \\
\hline Relative apical LS & $1.2 \pm 1.9$ & $1.3 \pm 0.7$ & $1.7 \pm 1.4$ & $1.5 \pm 0.7$ & $1.3 \pm 1.9$ \\
\hline Relative apical LS >1, n (\%) & $56(50)$ & $17(55)$ & $8(66)$ & $15(83)$ & $16(32)$ \\
\hline
\end{tabular}

Data are presented as mean \pm SD or $n(\%)$. sAS/ATTRwt-, severe aortic stenosis without wild-type transthyretin amyloidosis; No AS/ATTRwt+, wild-type transthyretin amyloidosis without aortic stenosis; mAS/ATTRwt+, wild-type transthyretin amyloidosis with moderate aortic stenosis; sAS/ATTRwt+, wild-type transthyretin amyloidosis with severe aortic stenosis; AVA, aortic valve area; iAVA, indexed aortic valve area; LVEF, left ventricular ejection fraction; LS, longitudinal strain; LV, left ventricular.

(Table 2 continued the next page.)

Intra- and inter-rater reliability were assessed by an intraclass correlation coefficient from 10 randomly selected studies, including 6 patients with atrial fibrillation, reanalyzed by 2 observers. Differences were considered statistically significant for $\mathrm{P}$ values of $<0.05$. All analyses were performed using standard statistical software, SPSS version 20 (SPSS Inc., Chicago, IL, USA).

\section{Results}

\section{Study Population}

One-hundred and eleven patients were prospectively included. The mean population age was $79 \pm 12$ years. Most of the patients $(74 \%)$ were male. No patient had transthyretin mutation after genetic testing and all patient were considered as having ATTRwt. No patients with severe AS without ATTRwt had monoclonal gammopathy. The population's baseline characteristics according to the presence of AS and/or ATTRwt are listed in the Table 1. Patients with ATTRwt were more likely to be male and had more atrial fibrillation than patients with AS alone.
Patients with both ATTRwt and AS were older. Patients with both AS (whatever the degree) and ATTRwt were more likely to have first degree atrioventricular block and/or intraventricular conduction delay than patients with ATTRwt without AS. Finally, patients without ATTRwt had higher systolic arterial blood pressure, particularly compared with patients who have ATTRwt without AS.

There were $9(50 \%)$ and $16(32 \%)$ patients with low flow - low gradient among patients with severe AS with and without ATTRwt, respectively $(\mathrm{P}=0.119)$. Among them, 3 $(33 \%)$ and $4(25 \%)$ had paradoxical low flow - low gradient AS, respectively. Among these latter patients, severe AS was confirmed by calcium scoring for all patients with ATTRwt and for $3(75 \%)$ patients without ATTRwt. Severe AS was confirmed with dobutamine stress echocardiography for 1 patient with low flow - low gradient without ATTRwt.

Regarding associated valvular diseases, there were 3 $(10 \%), 1(3 \%)$ and $1(3 \%)$ moderate aortic regurgitation, mitral stenosis and tricuspid regurgitation, respectively, among patients with ATTRwt without AS; 1 (8\%) moder- 


\begin{tabular}{|c|c|c|c|c|c|c|c|}
\hline & \multirow{2}{*}{$P$ value } & \multicolumn{6}{|c|}{ Post-hoc analysis } \\
\hline & & 1 vs. 2 & 2 vs. 3 & 3 vs. 4 & 1 vs. 3 & 1 vs. 4 & 2 vs. 4 \\
\hline \multicolumn{8}{|l|}{ General parameters } \\
\hline LVEF, \% & 0.296 & & & & & & \\
\hline LVEF <50\%, n (\%) & 0.262 & & & & & & \\
\hline LV end diastolic volume, $\mathrm{mL} / \mathrm{m}^{2}$ & 0.029 & 1.000 & 1.000 & 0.209 & 1.000 & 0.667 & 0.071 \\
\hline Stroke volume index, $\mathrm{mL} / \mathrm{m}^{2}$ & 0.602 & & & & & & \\
\hline LV septal wall thickness, $\mathrm{mm}$ & $<0.0001$ & 1.000 & 1.000 & 0.019 & 0.773 & $<0.0001$ & 0.001 \\
\hline LV posterior wall thickness, $\mathrm{mm}$ & 0.024 & 1.000 & 0.666 & 0.015 & 0.105 & 1.000 & 1.000 \\
\hline LV mass index, $\mathrm{g} / \mathrm{m}^{2}$ & $<0.0001$ & 1.000 & 0.149 & $<0.0001$ & 0.001 & 0.181 & 0.101 \\
\hline Wall motion score index & 0.449 & & & & & & \\
\hline \multicolumn{8}{|l|}{ Transmitral flow (\%) } \\
\hline Type 1 & 0.016 & 0.421 & 0.545 & 0.027 & 0.532 & 0.017 & 0.027 \\
\hline Type 2 & 0.729 & & & & & & \\
\hline Type 3/Exclusive E wave & 0.041 & 0.232 & 0.412 & 0.070 & 0.426 & 0.108 & 0.031 \\
\hline Lateral e' wave, $\mathrm{cm} / \mathrm{s}$ & 0.092 & & & & & & \\
\hline Left atrial volume, $\mathrm{mL} / \mathrm{m}^{2}$ & 0.666 & & & & & & \\
\hline \multicolumn{8}{|l|}{ Aortic valvular parameters } \\
\hline Mean transvalvular gradient, $\mathrm{mmHg}$ & $<0.0001$ & 0.007 & 0.001 & 0.101 & $<0.0001$ & $<0.0001$ & $<0.0001$ \\
\hline Mean transvalvular gradient $<40 \mathrm{mmHg}, \mathrm{n}(\%)$ & $<0.0001$ & 1.000 & 0.007 & 0.017 & $<0.0001$ & $<0.0001$ & $<0.0001$ \\
\hline $\mathrm{AVA}, \mathrm{cm}^{2}$ & $<0.0001$ & $<0.0001$ & 0.013 & 1.000 & $<0.0001$ & $<0.0001$ & 0.002 \\
\hline $\mathrm{iAVA}, \mathrm{cm}^{2} / \mathrm{m}^{2}$ & $<0.0001$ & $<0.0001$ & 0.032 & 0.455 & $<0.0001$ & $<0.0001$ & 0.005 \\
\hline Transvalvular peak velocity, $\mathrm{m} / \mathrm{s}$ & $<0.0001$ & $<0.0001$ & $<0.0001$ & 0.455 & $<0.0001$ & $<0.0001$ & $<0.0001$ \\
\hline Aortic valve velocity ratio & $<0.0001$ & $<0.0001$ & 0.010 & 1.000 & $<0.0001$ & $<0.0001$ & 0.006 \\
\hline \multicolumn{8}{|l|}{ Longitudinal strain parameters } \\
\hline Global longitudinal strain, \% & $<0.0001$ & 1.000 & 1.000 & 0.005 & 1.000 & 0.004 & 0.006 \\
\hline Mean basal longitudinal strain, $\%$ & $<0.0001$ & 1.000 & 1.000 & 0.001 & 1.000 & 0.011 & 0.022 \\
\hline Mean mid longitudinal strain, \% & $<0.0001$ & 1.000 & 1.000 & 0.007 & 1.000 & 0.005 & 0.023 \\
\hline Mean apical longitudinal strain, \% & 0.275 & & & & & & \\
\hline Relative apical LS & 0.538 & & & & & & \\
\hline Relative apical LS >1, n (\%) & 0.001 & 0.363 & 0.266 & $<0.0001$ & 0.041 & 0.036 & 0.031 \\
\hline
\end{tabular}

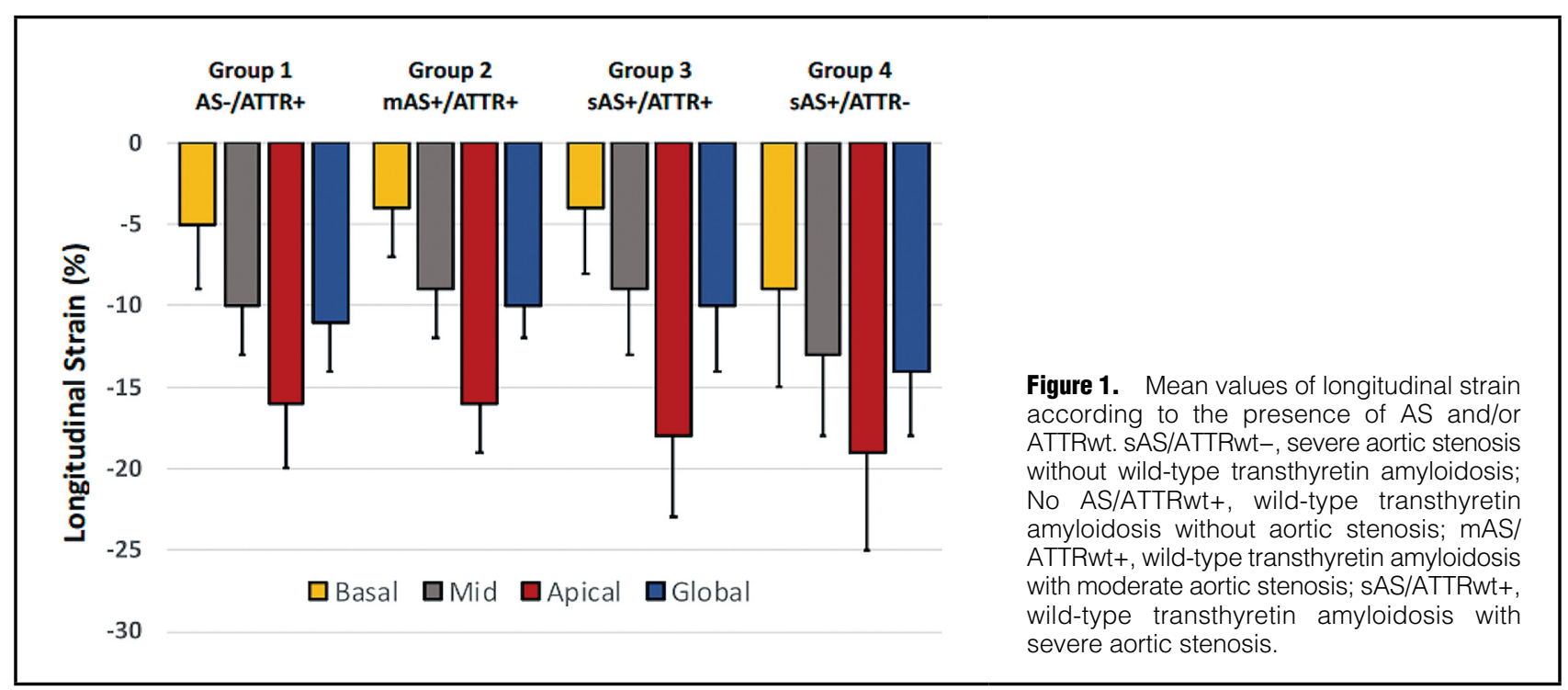


Whole population

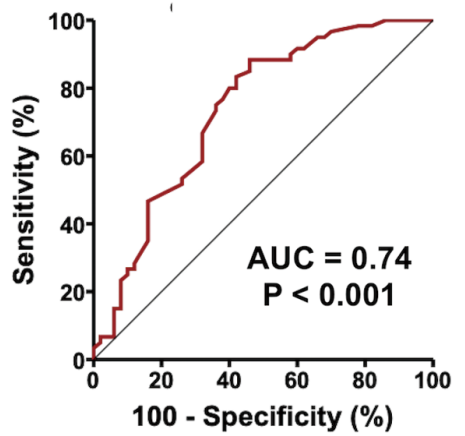

Aortic stenosis

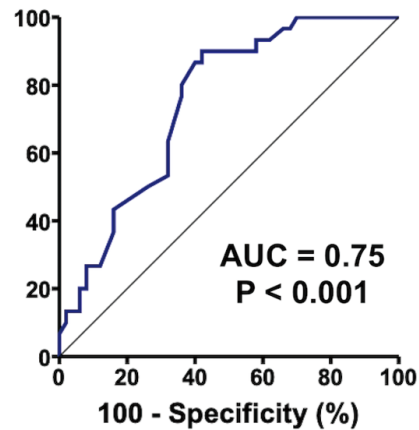

Severe aortic stenosis

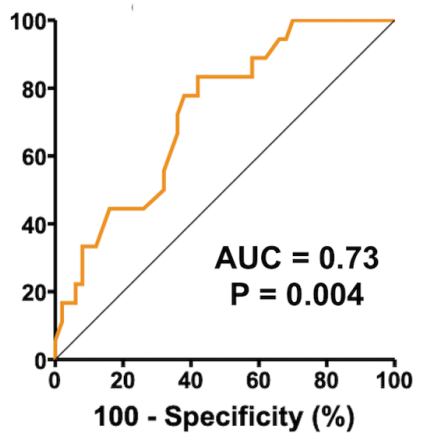

Figure 2. Receiver-operating characteristic curves of global longitudinal strain (LS) to predict ATTR among the whole population and according to the severity of AS. SAS/ATTRwt-, severe aortic stenosis without wild-type transthyretin amyloidosis; No AS/ ATTRwt+, wild-type transthyretin amyloidosis without aortic stenosis; mAS/ATTRwt+, wild-type transthyretin amyloidosis with moderate aortic stenosis; SAS/ATTRwt+, wild-type transthyretin amyloidosis with severe aortic stenosis. AUC, area under the receiver operating characteristic curve.

Whole population

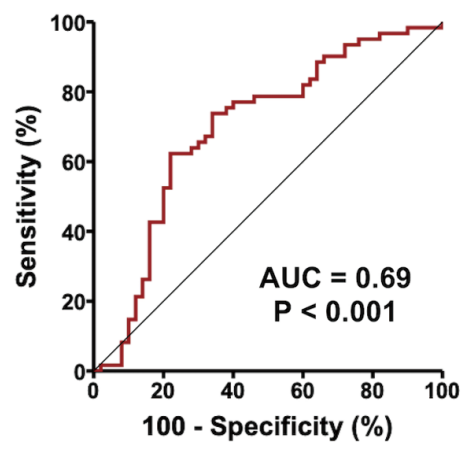

Aortic stenosis

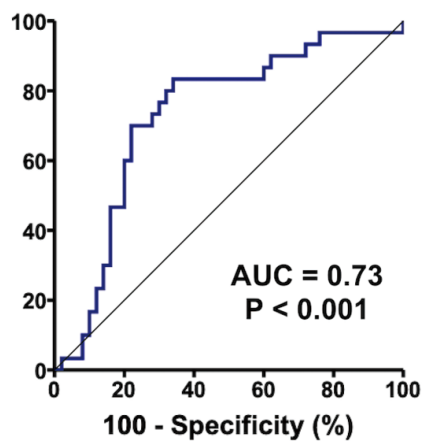

\section{Severe aortic stenosis}

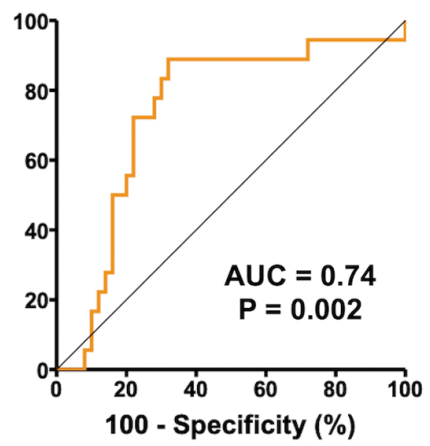

Figure 3. Receiver-operating characteristic curves of relative apical longitudinal strain (LS) to predict ATTR among the whole population and according to the severity of AS. SAS/ATTRWt-, severe aortic stenosis without wild-type transthyretin amyloidosis; No AS/ATTRwt+, wild-type transthyretin amyloidosis without aortic stenosis; mAS/ATTRwt+, wild-type transthyretin amyloidosis with moderate aortic stenosis; sAS/ATTRwt+, wild-type transthyretin amyloidosis with severe aortic stenosis. AUC, area under the receiver operating characteristic curve.

ate mitral regurgitation among patients with ATTRwt and moderate AS, no other valvular disease among patients with ATTRwt and severe AS; and $2(4 \%)$ and 2 (4\%) moderate mitral regurgitation and mitral stenosis, respectively, among patients with severe AS without ATTRwt.

\section{Echocardiographic Parameters}

Echocardiographic parameters are presented in the Table 2. Left ventricular ejection fraction was not different between patients with or without ATTRwt, even in the presence of AS. Diastolic dysfunction was more frequent in patients with ATTRwt despite no difference in left atrial volume or lateral Ea wave. Left ventricular mass index was greater in patients with ATTRwt, especially in patients with concomitant severe AS. Both mean transvalvular gradient and peak transvalvular velocity were lower in patients with severe AS when ATTRwt was present, despite no difference in aortic valve area. Relative apical LS was $>1.0$ in $32 \%$ of patients with severe AS without ATTRwt and $<1.0$ in $45 \%, 34 \%$ and $17 \%$ of patients with ATTRwt and without AS, with moderate AS and with severe AS, respectively. Global LS was lower in patients with ATTRwt as compared to patients without ATTRwt, even in the presence of severe AS. Wall motion score index was normal in $18(58 \%), 7(58 \%), 12(66 \%)$ and $36(72 \%)$ patients with ATTRwt without AS; ATTRwt and moderate AS, ATTRwt and severe AS, and severe AS without ATTRwt, respectively $(\mathrm{P}=0.576)$. All other patients had global hypokinesia, except for $1(3 \%)$ and $5(10 \%)$ patients among patients with ATTRwt without AS and patients who had severe AS without ATTRwt, respectively, and who had segmental wall motion abnormalities of 1 to 2 


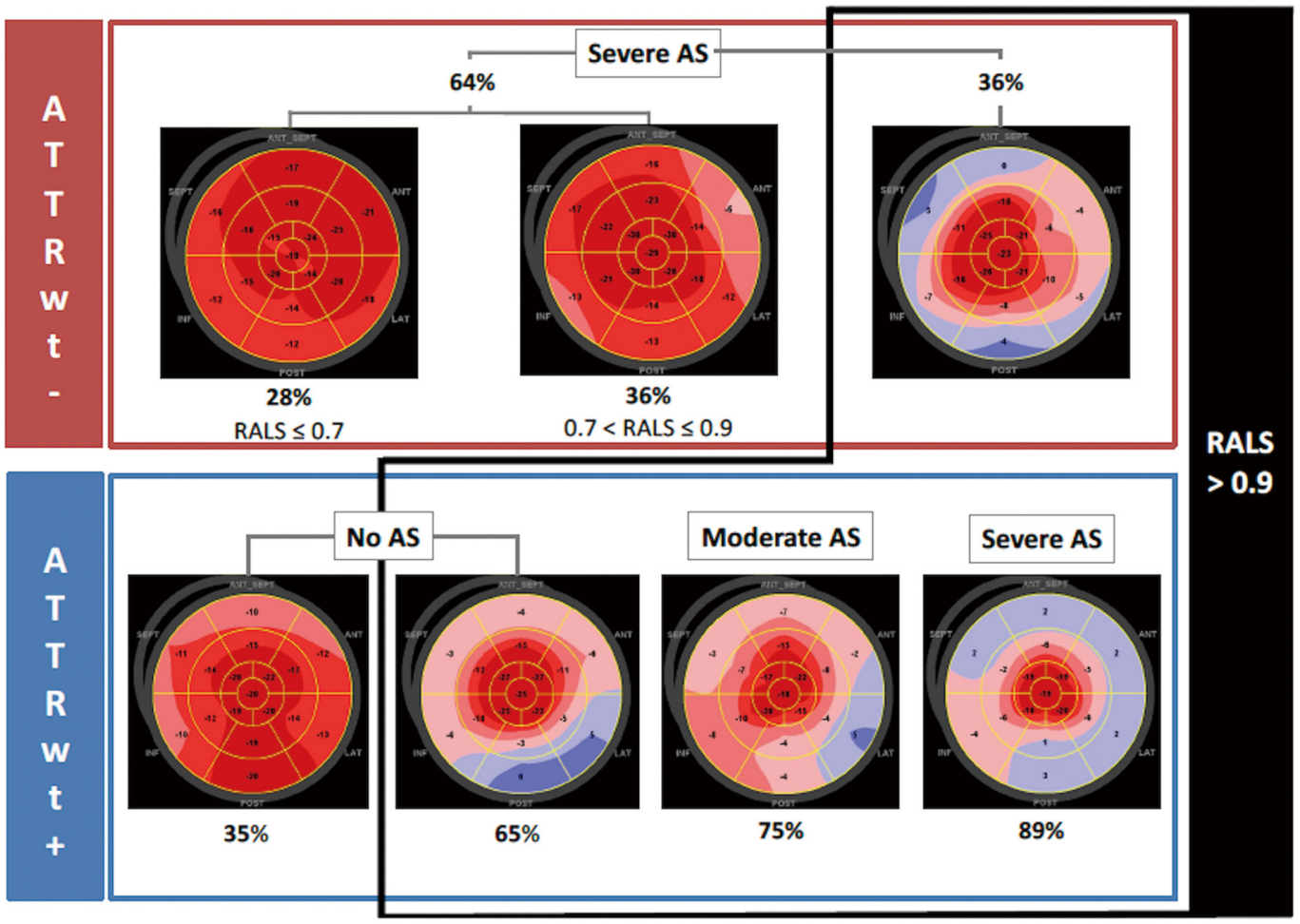

Figure 4. Proportion of significant relative apical longitudinal strain (RALS >0.9) among all subgroups considering AS and/or ATTRwt. ATTRwt-, patients without wild-type transthyretin amyloidosis; ATTRwt+, patients with wild-type transthyretin amyloidosis; AS, aortic stenosis; RALS, relative apical longitudinal strain.

segments $(\mathrm{P}=0.167)$. History of myocardial infarction had no impact on the results (data not shown).

Figure 1 shows the mean values of LS according to the presence of AS and/or ATTRwt. There was a progressive decrease in global LS from patients who had severe AS without ATTRwt to patients who had ATTRwt without AS and then, to patients with both ATTRwt and moderate, and then severe, AS. This decrease was similar for basal and mid LS. Apical LS was not different among groups.

The best cut-off value of global LS to identify ATTRwt cardiac amyloidosis among the whole population was $-15 \%$, allowing a sensitivity and specificity of $88 \%$ and $54 \%$, respectively (area under the ROC curve $0.74,95 \%$ confidence interval 0.64-0.83, $\mathrm{P}<0.001$; Figure 2). The best cut-off value of global LS to identify ATTRwt cardiac amyloidosis among patients with AS (whatever the severity) was $-14 \%$, allowing a sensitivity and specificity of $90 \%$ and $58 \%$, respectively (area under the ROC curve $0.75,95 \%$ confidence interval $0.64-0.85, \mathrm{P}<0.001$; Figure 2). The best cut-off value of global LS to identify ATTRwt cardiac amyloidosis among patients with severe AS was $-14 \%$, allowing a sensitivity and specificity of $83 \%$ and $58 \%$, respectively (area under the ROC curve $0.73,95 \%$ confidence interval 0.60-0.86, $\mathrm{P}=0.004$; Figure 2).

The best cut-off value of relative apical LS to identify ATTRwt cardiac amyloidosis among the whole population was 0.9 , allowing a sensitivity and specificity of $74 \%$ and $66 \%$, respectively (area under the ROC curve $0.70,95 \%$ confidence interval $0.59-0.80, \mathrm{P}<0.001$; Figure 3). The best cut-off value of relative apical LS to identify ATTRwt cardiac amyloidosis among patients with AS (whatever the severity) was 1.0, allowing a sensitivity and specificity of $77 \%$ and $70 \%$, respectively (area under the ROC curve 0.73 , $95 \%$ confidence interval $0.61-0.85, \mathrm{P}<0.001$; Figure 3 ). The best cut-off value of relative apical LS to identify ATTRwt cardiac amyloidosis among patients with severe AS was 1.0, allowing a sensitivity and specificity of $89 \%$ and $68 \%$, respectively (area under the ROC curve 0.74 , 95\% confidence interval 0.61-0.88, $\mathrm{P}=0.002$; Figure 3).

Relative apical LS $>0.9$ was identified in $36 \%$ of patients with severe AS despite absence of ATTRwt, 65\% of patients who had ATTRwt without AS, and finally $75 \%$ and $89 \%$ of patients who had ATTRwt and moderate or severe AS, respectively (Figure 4). Finally, 36\% of patients who had severe AS without ATTRwt had intermediate relative apical LS between 0.7 and 0.9 (Figure 4).

\section{Reproducibility}

For intra-rater reliability and inter-rater reliability of LS, intra-class correlation coefficients were 0.94 (95\% CI $0.78-$ $0.99 ; \mathrm{P}<0.001)$ and 0.94 (95\% CI 0.78-0.99; $\mathrm{P}<0.001)$, respectively.

\section{Discussion}

Our study shows that both cardiac ATTRwt and AS decrease global LS, but the decrease in global LS is particularly pronounced in the presence of an ATTRwt. A cut-off value 
of $-14 \%$ is sensitive, but not specific for identifying ATTRwt among patients with severe AS. The decrease of basal LS is common in patients with AS, and can lead to a relative sparing of LS in the left ventricular apex, even in the absence of ATTRwt. Consequently, in patients with severe AS, a cut-off value of 1.0 for the relative LS to identify cardiac amyloidosis is sensitive but less specific. In our population, the optimal cut-off value for the relative LS to identify cardiac amyloidosis was 0.9 , which is slightly below the value of 1.0 previously reported.11 However, despite this more permissive value, $35 \%, 25 \%$ and $11 \%$ of patients who had ATTRwt without AS, with moderate AS and with severe AS, respectively, did not reach this cut-off value. Coexistence of AS and ATTRwt is associated with an exacerbated apical longitudinal sparing pattern as a consequence of a major alteration in basal segments and, to a lesser extent, in median segments. This pattern is even more pronounced than in patients who have ATTRwt without AS.

These findings demonstrate the heterogeneity of patients with cardiac amyloidosis and suggest caution in the diagnostic algorithm, which requires combining clues. ${ }^{19}$ In their princeps publication, Phelan et al reported a sensitivity of $93 \%$ and a specificity of $82 \%$ for a relative apical LS of 1.0 in differentiating CA from controls (either AS or other cause of left ventricular hypertrophy). ${ }^{11}$ We found the same cut-off value for identifying patients with ATTRwt among patients with $\mathrm{AS}$, with a slight lower performance (sensitivity $77 \%$, specificity $70 \%$, AUC 0.73 for all patients with AS and sensitivity $89 \%$, specificity $68 \%$, AUC 0.74 for patients with severe AS). This discrepancy could be explained by a different population profile because their data were from a mixed population of light chains and ATTRwt patients.

Furthermore, it is important to note that, in our whole population including patients with and without AS, the cut-off value of relative apical LS was slightly lower (i.e., $0.9)$ for identifying patients with ATTRwt. There seems to exist an evolution of the relative apical sparing during the natural history of the disease. Indeed, amyloid fibrils settle preferentially at the base of the myocardium, ${ }^{20}$ explaining the progressive appearance of relative apical sparing of LS. However, it seems that this pattern is not constant and that other parameters could impact the LS for patients with ATTRwt. The study by Castano et al, which looked at the phenotype of patients with ATTRwt undergoing transcatheter aortic valve replacement, ${ }^{5}$ found overall similar results to our study; compared with patients without ATTRwt, patients with ATTRwt have a thicker interventricular septum, higher left ventricular mass index, lower mean transvalvular aortic gradient, more advanced diastolic dysfunction, more impaired global LS, but the same relative apical LS. Our population of patients with both AS and ATTRwt have the same age (i.e., 86 years), which is an elderly population whose co-morbidities may contribute to a decrease in the global LS and explain the disappearance of the relative apical LS. We suppose that several parameters influence the LS pattern and that with aging, the sensitivity of relative apical LS for identifying patients with ATTRwt decreases, which could explain why $35 \%$ of our population who have ATTRwt without AS was below 0.9.

Our results show a progressive alteration of LS from apex to base in all subgroups, including patients who have AS without ATTRwt, but it was more pronounced in patients with ATTRwt. It is well known that there is a natural gradient between the base and the apex; ${ }^{21}$ however, this gradient does not reach the value found in cardiac amyloidosis and that described by Phelan et al.11 In patients with amyloidosis, a decrease of basal LS has been showed to correlate with preferential amyloid fibril deposition in the basal segments. ${ }^{20}$ In patient who have AS without ATTRwt, impairment of LS especially in basal segments could be explained by the combination of chronic pressure overload due to AS and presence of mitral ring calcifications frequently associated with AS, particularly in the elderly. This could also partially explain the difference between our results and the previous results of Phelan et al, ${ }^{11}$ given that our population is $\sim 10$ years older than theirs. Previous series showed a predominant basal distribution of late gadolinium enhancement by cardiac magnetic resonance imaging in patients with severe $\mathrm{AS},{ }^{22}$ related to myocardial fibrosis due to chronic pressure overload, which was confirmed by pathology. ${ }^{23}$ This may explain that $36 \%$ of our patients who had severe AS without ATTRwt showed an intermediate decrease of relative apical LS (i.e., between 0.7 and 0.9). These results might encourage clinicians to search for ATTRwt in the presence of AS, whatever the severity, when associated with low global LS and particularly in the presence of a relative apical sparing.

More than an additive effect of AS and ATTRwt on LS, our study raises the difficult question of the possible interaction between aortic valve degeneration and myocardial amyloid deposition, as suggested by the high prevalence of transthyretin amyloid deposition in removed aortic valves, but with only few and limited echocardiographic data. ${ }^{\mathbf{2 4}}$ The realization of a diagnostic score, although necessary, was not feasible on these data alone. However, given the high prevalence of ATTRwt among patients with severe AS undergoing aortic valve replacement, either surgical ${ }^{4}$ or transcatheter, ${ }^{5,25}$ particularly in elderly males, ${ }^{5,26}$ given the worst prognosis carried by these patients ${ }^{25,26}$ and facing the lack of specificity of the relative apical LS and the global LS among this population, bone scintigraphy should be considered for patients aged $>80$ years who have severe AS with either a decrease of global LS $\geq-14 \%$ or relative apical LS $\geq 1.0$, particularly in the presence of conduction disorders or atrial fibrillation, or a clear increase in natriuretic peptides. Although MRI is interesting for the diagnosis of cardiac amyloidosis, ${ }^{27}$ but given the high prevalence of ATTRwt and the need to finally perform bone scintigraphy to confirm this diagnosis, bone scintigraphy should be considered from the outset, without MRI.

\section{Study Limitations}

First, this study has all the limitations associated with single site, but also small sample studies considering unequal size groups, especially those combining ATTRwt and AS. The small sample size of each subgroup encourages caution in interpreting the results. Indeed, larger subgroups would may have enabled more significant or relevant results for clinical practice. Indeed, it is important to note that the subgroups are not strictly comparable. Patients with both AS and ATTRwt are more likely to be male and older than the rest of the population. However, even if the size effects the hypothesis, our results suggest greater vigilance with regard to the diagnosis of cardiac amyloidosis in the population of patients with AS who are at high risk of ATTR, particularly in older males.

Second, we cannot exclude that myocardial ischemia 
may be involved in the observed strain pattern. However, it seems unlikely that myocardial ischemia would affect the basal segments circumferentially. In addition, our population reflects the population of daily clinical practice in which the ischemic status of patients is not known. Our study simply shows that, in this type of population, strain parameters can be misleading as to whether there is relative apical sparing of LS.

Center selection bias in the study population probably explains some of the discrepancies observed in comparison to previous studies. Amyloidosis is a heterogenous disease, with a wide spectrum of presentations and different degrees of clinical expression depending on infiltrative burden. Consequently, diagnostic tools should be validated on large multicenter cohorts to avoid center effects and selection bias.

\section{Conclusions}

Both cardiac ATTRwt and AS decrease global LS, but the decrease in global LS is particularly pronounced in the presence of an ATTRwt. Relative apical sparing of LS is common in patients with AS, even in absence of ATTRwt. Given the prognostic and therapeutic implications, transthyretin cardiac amyloidosis should be systematically sought using bone scintigraphy in the presence of AS, whatever the severity, when associated with low global LS and, particularly, in the presence of relative apical sparing. Absence of relative apical sparing of LS does not allow the ruling out of ATTRwt. Further studies are needed to refine the diagnosis of cardiac amyloidosis in the population of patients with AS, particularly given the physio-pathological links between these 2 diseases.

\section{Disclosures}

The authors have no conflicts of interest to disclose.

\section{IRB Information}

The present study was approved by the ethics committee for research of the University Hospital of Toulouse (Reference number: 02-815).

\section{References}

1. Lindroos M, Kupari M, Heikkila J, Tilvis R. Prevalence of aortic valve abnormalities in the elderly: An echocardiographic study of a random population sample. J Am Coll Cardiol 1993; 21: $1220-1225$.

2. Baumgartner H, Falk V, Bax JJ, De Bonis M, Hamm C, Holm PJ, et al. 2017 ESC/EACTS Guidelines for the management of valvular heart disease. Eur Heart J 2017; 38: 2739-2791.

3. Dayan V, Vignolo G, Magne J, Clavel MA, Mohty D, Pibarot P. Outcome and impact of aortic valve replacement in patients with preserved LVEF and low-gradient aortic stenosis. J Am Coll Cardiol 2015; 66: 2594-2603.

4. Treibel TA, Fontana M, Gilbertson JA, Castelletti S, White SK, Scully PR, et al. Occult transthyretin cardiac amyloid in severe calcific aortic stenosis: Prevalence and prognosis in patients undergoing surgical aortic valve replacement. Circ Cardiovasc Imaging 2016; 9: $\mathrm{e} 005066$.

5. Castano A, Narotsky DL, Hamid N, Khalique OK, Morgenstern $\mathrm{R}$, DeLuca A, et al. Unveiling transthyretin cardiac amyloidosis and its predictors among elderly patients with severe aortic stenosis undergoing transcatheter aortic valve replacement. Eur Heart J 2017; 38: 2879-2887.

6. Nietlispach F, Webb JG, Ye J, Cheung A, Lichtenstein SV, Carere RG, et al. Pathology of transcatheter valve therapy. JACC Cardiovasc Interv 2012; 5: 582-590.

7. Maurer MS, Schwartz JH, Gundapaneni B, Elliott PM, Merlini $\mathrm{G}$, Waddington-Cruz $\mathrm{M}$, et al. Tafamidis treatment for patients with transthyretin amyloid cardiomyopathy. N Engl J Med 2018; 379: $1007-1016$
8. Adams D, Gonzalez-Duarte A, O'Riordan WD, Yang CC, Ueda M, Kristen A V, et al. Patisiran, an RNAi Therapeutic, for hereditary transthyretin amyloidosis. N Engl J Med 2018; 379: $11-21$.

9. Benson MD, Waddington-Cruz M, Berk JL, Polydefkis M, Dyck PJ, Wang AK, et al. Inotersen treatment for patients with hereditary transthyretin amyloidosis. $N$ Engl J Med 2018; 379: 22-31.

10. Pagourelias ED, Mirea O, Duchenne J, Van Cleemput J, Delforge $\mathbf{M}$, Bogaert $\mathbf{J}$, et al. Echo parameters for differential diagnosis in cardiac amyloidosis: A head-to-head comparison of deformation and nondeformation parameters. Circ Cardiovasc Imaging 2017; 10: $\mathrm{e} 005588$.

11. Phelan D, Collier P, Thavendiranathan P, Popovic ZB, Hanna $\mathrm{M}$, Plana JC, et al. Relative apical sparing of longitudinal strain using two-dimensional speckle-tracking echocardiography is both sensitive and specific for the diagnosis of cardiac amyloidosis. Heart 2012; 98: 1442-1448.

12. Lee GY, Kim HK, Choi JO, Chang SA, Oh JK, Jeon ES, et al. Visual assessment of relative apical sparing pattern is more useful than quantitative assessment for diagnosing cardiac amyloidosis in borderline or mildly increased left ventricular wall thickness. Circ J 2015; 79: 1575-1584.

13. Wechalekar AD, Gillmore JD, Hawkins PN. Systemic amyloidosis Lancet (London, England) 2016; 387: 2641-2654.

14. Lang RM, Badano LP, Mor-Avi V, Afilalo J, Armstrong A, Ernande L, et al. Recommendations for cardiac chamber quantification by echocardiography in adults: An update from the American Society of Echocardiography and the European Association of Cardiovascular Imaging. Eur Heart J Cardiovasc Imaging 2015; 16: 233-270.

15. Nagueh SF, Smiseth OA, Appleton CP, Byrd BF 3rd, Dokainish $\mathrm{H}$, Edvardsen T, et al. Recommendations for the evaluation of left ventricular diastolic function by echocardiography: An update from the American Society of Echocardiography and the European Association of Cardiovascular Imaging. Eur Heart $J$ Cardiovasc Imaging 2016; 17: 1321-1360.

16. Baumgartner HC, Hung JCC, Bermejo J, Chambers JB, Edvardsen $\mathrm{T}$, Goldstein S, et al. Recommendations on the echocardiographic assessment of aortic valve stenosis: A focused update from the European Association of Cardiovascular Imaging and the American Society of Echocardiography. Eur Heart J Cardiovasc Imaging 2017; 18: 254-275.

17. Voigt JU, Pedrizzetti G, Lysyansky P, Marwick TH, Houle H, Baumann R, et al. Definitions for a common standard for 2D speckle tracking echocardiography: Consensus document of the EACVI/ASE/Industry Task Force to standardize deformation imaging. Eur Heart J Cardiovasc Imaging 2015; 16: 1-11.

18. Perugini E, Guidalotti PL, Salvi F, Cooke RMT, Pettinato C, Riva L, et al. Noninvasive etiologic diagnosis of cardiac amyloidosis using $99 \mathrm{mTc}$-3,3-diphosphono-1,2-propanodicarboxylic acid scintigraphy. J Am Coll Cardiol 2005; 46: 1076-1084.

19. Damy T, Maurer MS, Rapezzi C, Plante-Bordeneuve V, Karayal ON, Mundayat R, et al. Clinical, ECG and echocardiographic clues to the diagnosis of TTR-related cardiomyopathy. Open Heart 2016; 3: e000289.

20. Ternacle J, Bodez D, Guellich A, Audureau E, Rappeneau S, Lim P, et al. Causes and consequences of longitudinal LV dysfunction assessed by 2D strain echocardiography in cardiac amyloidosis. JACC Cardiovasc Imaging 2016; 9: 126-138.

21. Marwick TH, Leano RL, Brown J, Sun JP, Hoffmann R, Lysyansky $\mathrm{P}$, et al. Myocardial strain measurement with 2-dimensional speckle-tracking echocardiography: Definition of normal range. JACC Cardiovasc Imaging 2009; 2: 80-84.

22. Hoffmann R, Altiok E, Friedman Z, Becker M, Frick M. Myocardial deformation imaging by two-dimensional speckletracking echocardiography in comparison to late gadolinium enhancement cardiac magnetic resonance for analysis of myocardial fibrosis in severe aortic stenosis. Am J Cardiol 2014; 114: $1083-1088$.

23. Weidemann F, Herrmann S, Stork S, Niemann M, Frantz S, Lange V, et al. Impact of myocardial fibrosis in patients with symptomatic severe aortic stenosis. Circulation 2009; 120: $577-$ 584.

24. Kristen AV, Schnabel PA, Winter B, Helmke BM, Longerich T, Hardt $S$, et al. High prevalence of amyloid in 150 surgically removed heart valves: A comparison of histological and clinical data reveals a correlation to atheroinflammatory conditions. Cardiovasc Pathol 2010; 19: 228-235.

25. Scully PR, Treibel TA, Fontana M, Lloyd G, Mullen M, Pugliese $F$, et al. Prevalence of cardiac amyloidosis in patients referred for 
transcatheter aortic valve replacement. J Am Coll Cardiol 2018; 71: $463-464$

26. Cavalcante JL, Rijal S, Abdelkarim I, Althouse AD, Sharbaugh MS, Fridman Y, et al. Cardiac amyloidosis is prevalent in older patients with aortic stenosis and carries worse prognosis. J Cardiovasc Magn Reson 2017; 19: 98.

27. Syed IS, Glockner JF, Feng D, Araoz PA, Martinez MW, Edwards WD, et al. Role of cardiac magnetic resonance imaging in the detection of cardiac amyloidosis. JACC Cardiovasc Imaging 2010; 3: 155-164.

\section{Apendix}

On behalf of the Toulouse Amyloidosis Research Network collaborators: Laurent Alric, $\mathrm{MD}, \mathrm{PhD}^{\mathrm{a}}$; Léonardo Astudillo, $\mathrm{MD}^{\mathrm{b}}$; Christophe Bureau, $\mathrm{MD}, \mathrm{PhD}^{\mathrm{c}}$; Dominique Chauveau, $\mathrm{MD}, \mathrm{PhD}^{\mathrm{d}}$; Pascal Cintas, $\mathrm{MD}, \mathrm{PhD}^{\mathrm{e}}$; Audrey Delas, MD; Delphine Dupin-Deguine, MDg; Stanislas Faguer, $\mathrm{MD}, \mathrm{PhD}^{\mathrm{d}} ;$ Pauline Fournier, $\mathrm{MD}^{\mathrm{h}} ;$ Antoine Huart, MDd; Bénédicte Puissant, MDi; Grégory Pugnetb; Grégoire Prévot, MD; David Ribes, MD ${ }^{\mathrm{d}}$; Murielle Roussel, MD ${ }^{\mathrm{k}}$; Laurent Sailler, $\mathrm{MD}, \mathrm{PhD}^{\mathrm{b}}$ (a) Department of Internal Medicine and Digestive Diseases, Rangueil University Hospital, Toulouse, France

(b) Department of Internal Medicine, Toulouse University Hospital, Toulouse, France

(c) Department of Hepatology-Gastroenterology, Rangueil University Hospital, Toulouse, France

(d) Department of Nephrology and Referral Center for Rare Diseases, Rangueil University Hospital, Toulouse, France

(e) Department of Neurology, Purpan University Hospital, Toulouse, France

(f) Department of Pathology, IUCT Oncopôle, Toulouse, France

(g) Department of Genetic, Toulouse University Hospital, Toulouse, France

(h) Department of Cardiology, Rangueil University Hospital, Toulouse, France

(i) Immunology Laboratory, Toulouse University Hospital, Toulouse, France

(j) Department of Pneumology, Toulouse University Hospital, Toulouse, France

(k) Department of Hematology, Toulouse University Hospital, Toulouse, France 